\title{
Corrugated tube protocol for autogenous shrinkage measurements: review and statistical assessment
}

\author{
Mateusz Wyrzykowski $($ D $\cdot$ Zhangli Hu $\cdot$ Sadegh Ghourchian • \\ Karen Scrivener • Pietro Lura
}

Received: 29 March 2016/Accepted: 8 July 2016/Published online: 17 August 2016

(C) RILEM 2016

\begin{abstract}
Among different methods of measuring early age autogenous deformation of cementitious materials, the corrugated tube method described in the ASTMC1698-09 standard is one of the most commonly used. Despite the standardization and the common application of this method in the research community, only limited information has been published about the method's precision. This paper is dedicated to a review of this method, together with a statistical assessment of the results of an experimental study on three cement pastes. The autogenous deformation of cement pastes of water-to-cement ratio $0.30-0.36$, with and without internal curing, was measured by different operators in a single laboratory following the standard ASTM procedure. In addition to determining the method's precision, key factors influencing the results (i.e., rotation, temperature, time-zero) were identified and their effects were quantified. It was found that the method is sensitive
\end{abstract}

M. Wyrzykowski ( $\square) \cdot$ S. Ghourchian · P. Lura

Empa, Swiss Federal Laboratories for Materials Science

and Technology, Dübendorf, Switzerland

e-mail: Mateusz.Wyrzykowski@empa.ch

Z. Hu $\cdot$ K. Scrivener

Laboratory of Construction Materials, École

Polytechnique Fédérale de Lausanne, Lausanne,

Switzerland

S. Ghourchian · P. Lura

Institute for Building Materials, ETH Zurich, Zurich,

Switzerland enough to resolve different levels of shrinkage and its evolution in time, and that the effect of the operator is not significant. However, considerably high scatter was found between replicate samples within a single operator. The major source of the scatter was due to the inaccuracies in the initial measurement after setting (time-zero), being the reference point for strain determination.

Keywords Autogenous shrinkage - Cement . Corrugated tube method $\cdot$ High performance concrete . Precision

\section{Introduction}

Autogenous shrinkage of cement paste is one of the major causes of early-age cracking of high performance concrete (HPC) [1, 2]. Due to its practical importance, a number of experimental studies have been devoted to quantify it in the recent years, e.g. [3-7]. However, due to the fact that autogenous deformation needs to be distinguished from drying shrinkage or thermal deformations that may occur in cement-based materials at early ages, the choice of an appropriate measuring technique is all but non-trivial.

The different methods applied for measuring autogenous shrinkage of cement-based materials can be divided into volumetric and linear methods [3, 8-10]. It has been shown [3] that, assuming isotropy of 
autogenous shrinkage deformations after setting, the two types of methods can deliver equivalent results when sources of error and measurements artefacts are eliminated.

Since autogenous shrinkage is especially pronounced at early ages, when both hydration and selfdesiccation proceed at the highest rates [7], it is of crucial importance to follow the deformations starting already early on. This is why traditional shrinkage measurements on samples that require demolding prior to the measurements are not appropriate for assessing autogenous shrinkage. A number of different linear methods enabling measurements of autogenous shrinkage from casting were compared in a round robin study [10]. One of the most common approaches to determine linear autogenous shrinkage of cement paste from the time of casting was proposed originally by Jensen and Hansen [11]. They introduced a method where a cement paste is poured into a corrugated plastic tube. The tube is next sealed and placed horizontally on a rigid metal frame. The tube is considerably stiffer in the radial than in the longitudinal direction, therefore it enables measuring the linear shrinkage by following the displacement of the tube's ends. This can be conveniently done with digital displacement transducers attached to either both ends or only one end of the sample (with the other end fixed in the frame), or by using non-contact sensors, e.g. [12]. The tubes can be further placed in an oil bath which allows to control their temperature and further limits the evaporation through the necessarily imperfectly hermetic tube.

Later, the standard method for measuring autogenous shrinkage of cement pastes and mortar ASTM C1698-09 [13] was developed based on the original design by Jensen and Hansen [11]. According to that standard, measurements are performed manually on the corrugated tubes and only at a certain time steps (usually from every couple of hours in the beginning to every day or more up to a few weeks from casting). Even though the corrugated tube protocol allows measuring deformations already in the plastic state, the nature of standard measurement requires that the samples are moved and placed on a rigid frame equipped with a displacement transducer to perform each measurement. This is advantageous compared to the automatic measurements, since it allows measuring a number of samples by placing them sequentially on one frame equipped with only one displacement transducer. On the other hand, this economical advantage goes together with sacrificing the accuracy, since additional errors are induced by repositioning the sample before each sequential measurement takes place and by random changes in the position at which the sample is contacted by the transducer. Even more importantly, handling the samples according to the standard requires that the samples must be stiff enough to be moved and placed on the measuring frame without breaking them. Damaging of the fragile sample during handling may lead to invalid measurements [14]. Based on this limitation, the standard defines final set (measured with Vicat needle) as the starting point of the measurement. However, this brings in another source of inaccuracy, the determination of the setting time. If the autogenous measurement is started too early, handling of the sample, even if does not lead to any visible sample damage, may affect its length (since the material is very soft at that time). Last but not least, the time instant at which the initial measurement is taken, and consequently the determination of the initial sample length to which the strains are referenced, will have a paramount influence on the magnitude of determined strains. The last issue is related to the so-called time-zero, a key factor in the determination of autogenous shrinkage that has been extensively discussed in the literature (e.g. [15-19]).

The corrugated tube method has been applied for analysis of autogenous deformations of cement-based material, and the major factors influencing the measurements have been studied in e.g. [5, 10, 14, 20]. The most significant factors that may influence the measurements have been identified: temperature effects, bleeding and reference time; these factors are also analysed here. However, to the knowledge of the authors, there exists no systematic study quantifying the method's precision. This paper is aimed at filling this gap.

This paper provides a statistical assessment of ASTM C1698-09 method [13] and reviews its critical points. The method was analysed by performing manual measurements of autogenous deformations of cement pastes according to the standard and comparing them to the results of automatic measurements with a setup similar to that originally proposed in [11]. The standard measurements were performed by 4 different operators (in one single laboratory) in order to assess the sensitivity of the method upon different factors. The statistical analysis was 
performed using a univariate repeated measurements analysis of variance (RM ANOVA) [21]. The precision of the method was estimated with the betweenoperator reproducibility standard deviation based on the ISO 5725-2 standard [22]. Finally, the influence of the sample temperature, sample rotation and setting time was discussed. The tests were performed on cement pastes of w/c of $0.30,0.33$ and 0.36 , with and without internal curing by means of superabsorbent polymers (SAP) [23]. Internal curing was applied to obtain a wide range of deformation behaviour (from large shrinkage in the reference paste to moderate shrinkage or even expansion with internal curing), while at the same time minimizing the effect of bleeding, that would unavoidably occur in higher w/c cement pastes without internal curing [24].

\section{Materials and methods}

\subsection{Materials}

Three different cement pastes were used in the study, with the aim to follow three different levels of autogenous deformation. The $\mathrm{w} / \mathrm{c}$ of the cement pastes were: $0.30,0.33,0.36$, respectively, and the w/c 0.33 and 0.36 pastes had additionally SAP added as internal curing agent (with the entrained w/c equal to 0.03 and 0.06 , respectively [23]). Solution-polymerized SAP with particles of irregular shapes and sizes 63-125 $\mu \mathrm{m}$ and absorption $17 \mathrm{~g} / \mathrm{g}$ (determined by polished section analysis in [25]) were used.

The cement used was CEM I $42.5 \mathrm{~N}$ with the following oxide composition (by mass percent): $\mathrm{CaO}$ 64.1, $\mathrm{SiO}_{2}$ 19.7, $\mathrm{Al}_{2} \mathrm{O}_{3} 4.9, \mathrm{Fe}_{2} \mathrm{O}_{3} 2.9, \mathrm{SO}_{3} 2.9, \mathrm{MgO}$ 1.9. Deionized water was used as mixing water. A polycarboxylate-based superplasticizer was used in amount $0.3 \%$ (by mass of cement) for the w/c 0.30 cement paste and $0.5 \%$ for the w/c 0.33 and w/c 0.36 cement pastes to obtain enough flowability for filling the corrugated tubes. The amount of superplasticizer was accounted for in the total mixing water.

\subsection{Sample preparation}

Each paste was mixed using a 5-1 Hobart mixer and the volume of a mixing batch was about 11 . First, dry cement and SAP (where applied) were mixed in the dry state for $2 \mathrm{~min}$. Next, water was slowly added and wet mixing lasted for $5 \mathrm{~min}$ (with an intermediate $30 \mathrm{~s}$ pause for scraping of the mixing bowl).

Before filling each tube, it was closed at one end with an end plug and placed vertically in a steel pipe to provide support during filling. The steel pipe was fixed to a vibrating table. Directly after mixing, approximately $500 \mathrm{~g}$ of a paste was poured into a corrugated tube within about 2 min while applying vibrations. The tube was next closed with a second end plug while still in the vertical position. After filling all three tubes from a given mix, the tubes were cleaned from any spills of cement paste. In order to provide better sealing and minimize possible drying of the specimens or absorption of the oil in the case of storage in oil bath, a paraffin film was wrapped around the ends of the tube where it was closed by the end plugs. Next, adjustable hose clamps of diameter 20-25 mm were fastened at each end of the tube. This step, although not prescribed by the standard, was found necessary since it was found that some corrugated tubes crack at the ends. This is most likely due to the combined effect of stretching a tube by a sometimes slightly too large end plug and due to imperfect welding of a tube in a production process. If this happens, the end plug loosens and the specimen needs to be discarded. Using the hoses at the ends allowed completely eliminating such risk.

Four operators were involved in the study: operators 1 and 2 were experienced operators (with a couple to several years of experience and several tests performed previously), while operators 3 and 4 were trained immediately before the study and supervised by an experienced operator (operator 1). Each operator mixed individually one batch of each of the three pastes just before preparing the samples. The three pastes were prepared by an operator within a time window of about $3 \mathrm{~h}$. All samples were prepared by all operators within a time window of approximately 3 months, with a few weeks between the actions of each operator. All operators used the same batch of raw materials and corrugated tubes.

\subsection{Autogenous shrinkage measurements methods}

Two types of measurements were performed: (1) measurements according to the standard [13] and referred to as standard measurements; (2) measurements in which the samples were left untouched by an operator during the measuring period and the length 
was automatically measured at 1 min intervals by LVDTs, referred to as automatic measurements.

Standard measurements were taken initially at final set determined with Vicat needle and later at different time intervals. Before final set, the specimens were stored horizontally on corrugated aluminium sheets close to the measuring rigid frame. Before each measurement, a specimen was carefully placed on a rigid stainless steel frame, Fig. 1, and adjusted to reach approximately the same position (indicated by a mark painted on a sample plug, see [13]) while the reading was taken. A plastic support (a halved pipe) was delicately placed underneath a specimen to carefully move it to the measuring frame and minimize the risk of damage. At later ages, the sufficiently hard specimens were transported by lifting with two hands. Specimens undergoing automatic measurements were placed on a rigid frame approximately $1 \mathrm{~h}$ after the start of mixing. The exception concerns the specimens that were rotated before the automatic measurements until final setting time, and only at that point placed on a rigid frame for standard measurements or automatic measurements.

The reference length bar made of INVAR alloy used for calibrating the measuring bench had length of $425 \mathrm{~mm}$, Fig. 1.

Each standard measurement was performed on the same rigid frame equipped with a digital displacement transducer of $0.001 \mathrm{~mm}$ resolution (which would result in about $3 \mu \mathrm{m} / \mathrm{m}$ strain resolution), Fig. 1 . The

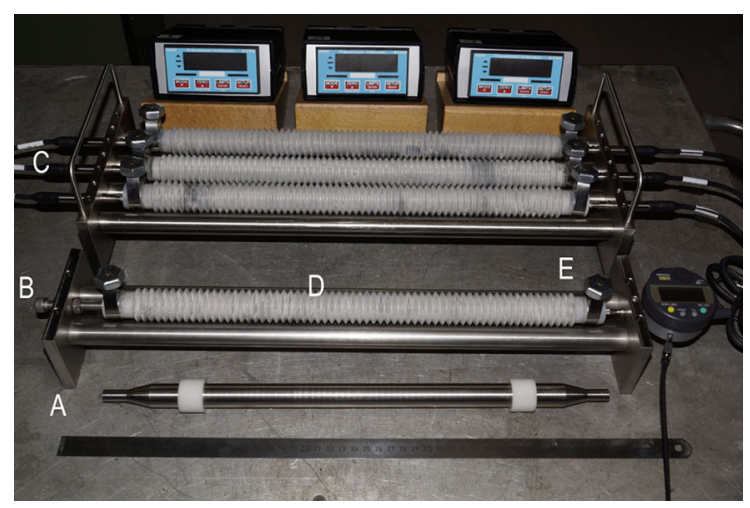

Fig. 1 Setups used for the measurements of autogenous deformations. $A$ reference bar, $B$ frame used for standard measurements equipped with digital deformation transducer, $C$ frame used for automatic measurements equipped with two LVDTs per sample, $D$ specimen: cement paste enclosed in corrugated tube, $E$ hose clamp used to tighten the closing of a tube (description in the text) transducer was zeroed by each operator before measuring each mixture (i.e. before each 3 -specimens' set).

All automatic measurements were performed on a rigid stainless-steel frame capable of accommodating three specimens at a time, Fig. 1. The frame was equipped with two LVDTs of resolution $0.0001 \mathrm{~mm}$, one at each end of the sample. The data was logged at $60 \mathrm{~s}$ intervals and the measurements lasted up to 7 days from water addition. The LVDTs were zeroed at the beginning of each 7-days' measuring period. The frame for automatic measurements was immersed in a bath filled with silicone oil (Rhodorsil 47V20). A circulating pump and a thermostat system allowed controlling the oil temperature at $19.8 \pm 0.1{ }^{\circ} \mathrm{C}$. In addition, silicone oil allows limiting the drying of the samples through the corrugated tubes that may not be perfectly impermeable for long-time measurements. At the same time, no penetration of the oil into the tubes could be observed, and no considerable mass increase due to oil absorption occurred through the measurement time.

All measurements were run and the samples were stored in the same climate-controlled room at $19.3 \pm 0.1{ }^{\circ} \mathrm{C}$ and $70 \pm 3 \% \mathrm{RH}$. In order to investigate the effect of sample rotation (which is recommended in the standard when measuring on higher w/c systems [13]), part of the samples were rotated around their longitudinal axis at $2 \mathrm{rpm}$ from casting to final setting time.

\subsection{Setting time}

Final set determined with the Vicat needle method was assumed as time-zero for the autogenous shrinkage measurements [13, 19]. A needle of $1 \mathrm{~mm}$ diameter and a mass of $300 \mathrm{~g}$ was used and final set was considered as the moment where the needle did not penetrate the sample any longer. The needle was automatically released at $5 \mathrm{~min}$ intervals. The measurement method followed in general the standard [26], with a modification concerning covering of the samples during the tests. This was necessary in order to provide conditions close to autogenous in terms of avoiding moisture exchange with the environment. For this reason a stretched food-wrap was placed over the cement paste surface (without touching it). Setting times were determined on 3 samples, each from independent mixings. The samples had the form of 
truncated cones with 75 and $85 \mathrm{~mm}$ diameters and $40 \mathrm{~mm}$ height enclosed in PVC moulds (changing from 16 to $6 \mathrm{~mm}$ in thickness along the height). Additionally, the temperature was measured in three samples by means of two thermocouples per sample. The thermocouples were placed at the bottom of each sample and about $2 \mathrm{~mm}$ below the top surface.

\subsection{Temperature measurements}

The evolution of the sample temperature inside corrugated tubes was measured on companion specimens filled with w/c 0.30 cement paste by means of thermocouples embedded in the central part of the corrugated tubes. Three samples were stored in the same climate-controlled room where all standard measurements were performed (at $19.3 \pm 0.1{ }^{\circ} \mathrm{C}$ ) and three samples were stored in the silicone oil bath where the automatic measurements were performed (at $19.8 \pm 0.1^{\circ} \mathrm{C}$ ). In both cases the requirements of the standard regarding ambient temperature control with a tolerance of $\pm 1.0{ }^{\circ} \mathrm{C}$ were fulfilled. The temperature in the samples and outside them (either in the air or in the oil bath) was measured by three thermocouples for each condition for 4 days at $1 \mathrm{~min}$ intervals at $\pm 0.1{ }^{\circ} \mathrm{C}$ resolution (the presented results are the average and the standard deviation).

\subsection{Statistical data assessment}

All obtained datasets were analysed using the analysis of variance (ANOVA) [27]. The aim of the analysis was to determine whether the method allows for finding differences between different mix compositions (w/c 0.30, w/c 0.33 and w/c 0.36) and following the differences in time. As a consequence, the fixed effect factors analysed were mix composition and age.

An important feature of the experiment design is that the set of measurements over time were performed on a set of the same subjects (specimens-corrugated tubes), which can reveal a certain similarity, especially the measurements performed close in time. Such design is referred to as a longitudinal study, and was statistically assessed by means of the repeated measurements ANOVA (RM ANOVA) [21, 27] with the Greenhouse-Geisser correction (for details see [21]).

The following univariate RM ANOVA models were analysed with mixed fixed and random effects:
(1) the measurements on three specimens of a given mix composition by each operator were aggregated (averaged) into one value and the random effects were assigned to the operator only;

(2) the random effects were assigned to individual specimens;

(3) a combined model, where the random effects were assigned to the individual specimens, with an additional blocking factor (assumed as fixed) due to the operator.

The three models can be presented symbolically as, respectively:

$$
\begin{aligned}
y_{i j k}= & \mu+\alpha_{i}+\beta_{j}+(\alpha \beta)_{i j}+\gamma_{k} \\
& +(\alpha \gamma)_{i j k}+(\beta \gamma)_{i j k}+(\alpha \beta \gamma)_{i j k}+e_{i j k} \\
y_{i j l}= & \mu+\alpha_{i}+\beta_{j}+(\alpha \beta)_{i j}+\delta_{l}+(\beta \delta)_{j l}+e_{i j l} \\
y_{i j k l}= & \mu+\alpha_{i}+\beta_{j}+\gamma_{k}+(\alpha \beta)_{i j}+\delta_{l}+(\beta \delta)_{j l}+e_{i j k l}
\end{aligned}
$$

where $y_{i j k}$ strain measured by operator $k$ at age $j$ for mix composition $i$ (average from three specimens), $y_{i j l}$ strain measured on the $l$ th specimen at age $j$ for mix composition $i, y_{i j k l}$ strain measured by operator $k$ at age $j$ for mix composition $i$ on the $l$ th specimen, $\mu$ common level (here assumed as zero strain), $\alpha_{i}$ effect of mix composition $i$ (fixed effect, between-subject factor), $\beta_{j}$ effect of age $j$ (fixed effect, within-subject factor), $\gamma_{k}$ effect of operator $k$ (fixed or random), $\delta_{l}$ effect of specimen $l$ (random, normally distributed with zero mean), $e$ error (random, normally distributed with zero mean)

The multiplication terms represent the interactions between different effects (random if any of the terms is random). For further reference on the ANOVA models and the used notation convention please refer to the statistical literature, e.g. [27].

The null-hypothesis of the performed test was that there are no differences between the sample mean at the level of a factor (different mix compositions or ages, and operators for model 3) and the overall mean. The null-hypothesis is rejected at a chosen significance level (assumed as 0.05 ) based on an $F$-test, where the variability between groups is compared to the variability within groups [27].

In addition to the RM ANOVA analysis described above, the accuracy characteristics were calculated in accordance with the ISO 5725-2 standard [22]. The 
standard considers two characteristics as a measure of accuracy: trueness and precision. Trueness requires knowledge of an accepted reference value, which is not applicable in the case considered here. Precision refers to the scatter of the test results and depends only on random errors. Precision can be further considered for two extreme types of conditions, namely repeatability conditions and reproducibility conditions. Repeatability conditions refer to the experiment run at the same conditions by the same operator on the same equipment in a short time window. Another extreme case is referred to as reproducibility conditions, when the aforementioned factors change and therefore contribute to the variability of the results. In the particular case applied here, in which only the operator and the tested material (different mixing and different samples) change and the measurements are run at different times, but the device and other conditions remain unvaried, intermediate conditions apply. Repeatability precision was assessed based on the measurements on different samples from one mixing treated as replicates and was calculated as a pooled within-operator standard deviation $s_{\mathrm{r}}$ [22]. Between-operator standard variation $s_{\mathrm{O}}$ was next calculated in a way similar to the between-laboratory standard deviation in accordance with [22]. Finally, an estimate of reproducibility precision between different operators was calculated from the sum of variances $s_{\mathrm{OR}}^{2}=s_{\mathrm{O}}^{2}+s_{\mathrm{r}}^{2}$.

\section{Results}

\subsection{Standard measurements}

Both the standard and the automatic measurements were zeroed at final set determined with the Vicat needle. The setting time results are presented in Table 1. Notice that the temperature in the w/c 0.30

Table 1 Setting time determined with Vicat needle (average \pm standard deviation, rounded to the closest $5 \mathrm{~min}$ )

\begin{tabular}{lll}
\hline Mix & Initial set & Final set \\
\hline w/c 0.30 & $3 \mathrm{~h} 45 \pm 10 \mathrm{~min}$ & $5 \mathrm{~h} 20 \pm 10 \mathrm{~min}$ \\
w/c 0.33 & $4 \mathrm{~h} 50 \pm 25 \mathrm{~min}$ & $6 \mathrm{~h} 30 \pm 5 \mathrm{~min}$ \\
w/c 0.36 & $5 \mathrm{~h} 15 \pm 20 \mathrm{~min}$ & $6 \mathrm{~h} 25 \pm 5 \mathrm{~min}$ \\
\hline
\end{tabular}

cement paste samples used for measuring setting time increased to $22.5 \pm 0.2$ and $23.3 \pm 0.2{ }^{\circ} \mathrm{C}$ at initial and final set, respectively.

At the time of Vicat-determined final set, the temperature of the w/c 0.30 cement paste inside the corrugated tubes stored in air was equal to $20.8 \pm 0.1{ }^{\circ} \mathrm{C}$. The maximum temperature was $22.8 \pm 0.1{ }^{\circ} \mathrm{C}$ and was recorded at approximately $15 \mathrm{~h}$ from start of mixing. After the peak, the temperature gradually decreased to equilibrate with the ambient temperature at the age of approximately 2.5 days. The maximum difference between the ambient air and sample temperature was equal to $3.5^{\circ} \mathrm{C}$.

The mass loss from the samples was also controlled in parallel to the length measurements. It is assumed in the standard [13] that all mass change is due to exchange of moisture between cement paste and the environment through a possibly permeable tube or end plugs. Measuring mass loss is therefore required in order to estimate the contribution of drying shrinkage caused by drying of the cement paste. The standard roughly estimates based on [28] that $1 \mathrm{~g}$ of water loss from the cement paste filling the tube (around $500 \mathrm{~g}$ in the tests performed here) should correspond to $200 \mu \mathrm{m} / \mathrm{m}$ of shrinkage. If the estimated shrinkage due to water loss at the conclusion of the test reaches more than $5 \%$ of the total measured strain, the test is considered invalid according to the standard. In the tests performed here, the maximum mass loss was equal to $0.09 \mathrm{~g}$ (and on average $0.04 \mathrm{~g}$ for all operators and all specimens), therefore around $0.02 \%$ of the mass of cement paste in the tube and around $0.07 \%$ of the total water content in the paste.

Following the estimate prescribed by the standard, $0.09 \mathrm{~g}$ should correspond to $18 \mu \mathrm{m} / \mathrm{m}$. Even though such strain due to drying seems to be negligible (it is considerably lower than the repeatability precision discussed later), it may be relatively high for some low-shrinkage mixtures. In the case of w/c 0.30 cement paste, $18 \mu \mathrm{m} / \mathrm{m}$ corresponds to at most $2 \%$ of total strain. However, for the w/c 0.36 paste with very low absolute strain, the contribution becomes much higher, around $25 \%$ of total shrinkage. It needs to be noted that the criterion of allowed $5 \%$ strain contribution due to drying is not relevant in case when the strain at conclusion of the test happens to be close to zero. A better criterion in such case could be defined at an absolute level of allowable error, e.g. related to the 
precision of the method or based on a relative error, but related to a maximum difference between the triplicate samples rather than to an absolute measured strain.

In Fig. 2 the results of standard measurements are presented as obtained by different operators. It can be seen that the general trends as well as the magnitudes were captured with a good agreement between the different operators. The ranking from highest to lowest shrinkage corresponds to the w/c used, with shrinkage increasing for decreasing w/c. The w/c 0.30 paste experienced the highest shrinkage rate during the first week of hardening and had the highest shrinkage at the age of 28 days. For the two pastes with internal curing by means of SAP, autogenous shrinkage was either strongly limited (w/c 0.33 ) or completely eliminated (w/c 0.36). These results are in line with the commonly observed trends in cement pastes with internal curing, e.g. [29-31].
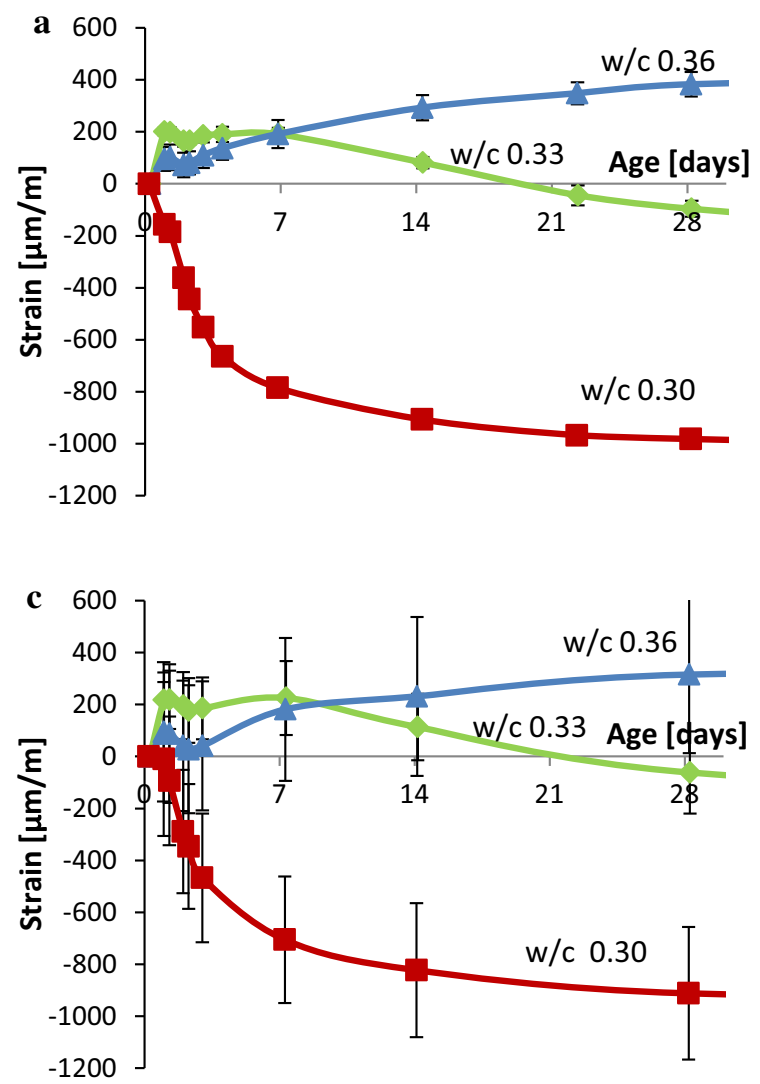

Fig. 2 Autogenous deformations of cement pastes according to standard measurements determined by operators 1-4 (a-d, respectively). The markers represent average results from three
A high scatter of the results within one operator resulting from testing different specimens is evident for the unexperienced operators 3 and 4 , see standard deviation bars in Fig. 2c, d, respectively. The variance does not change considerably in time but is approximately constant for each set of samples (each mix composition). It is therefore clear that the scatter between the different samples from one mix composition by one operator is mostly due to the initial measurement of specimens' lengths (time-zero measurements). This issue is further discussed in Sect. 3.

In Fig. 3 the deformations are presented for each mix obtained as the average from all operators. The error bars correspond to the standard deviation between the average values of each of the four operators. It can be seen in Fig. 3 that the scatter of the average strains between different operators was lower than the scatter of the individual samples for operators 3 and 4, (see Fig. 2c and d).
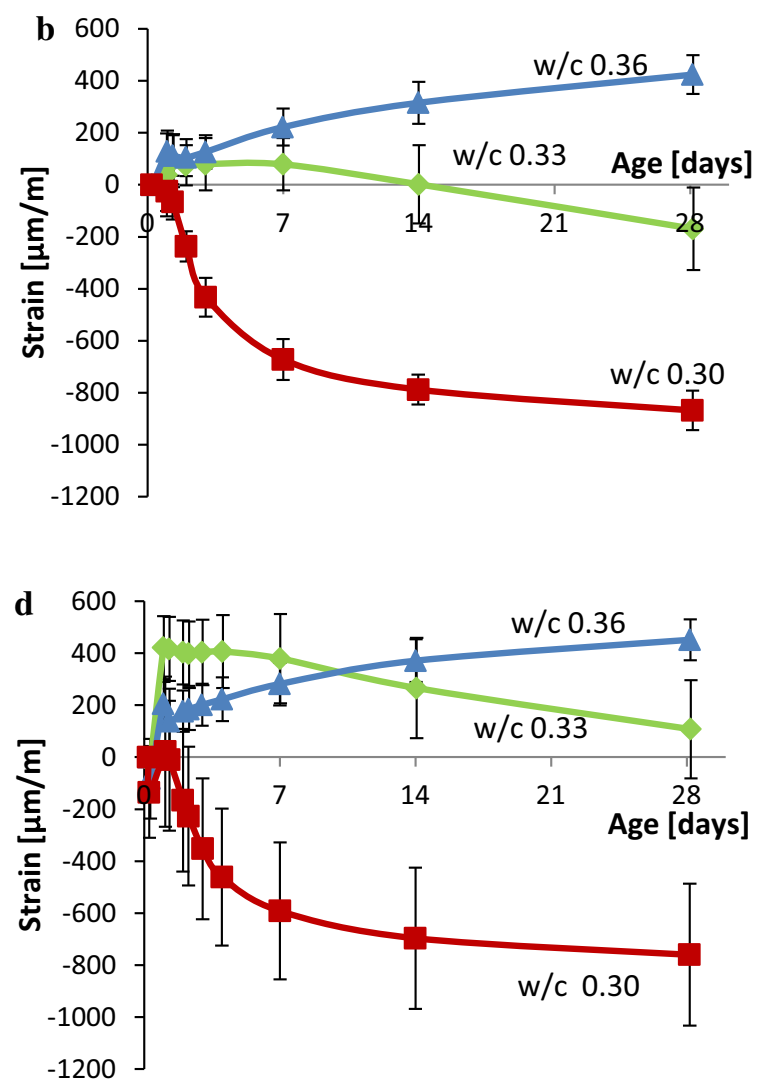

specimens from one mix/operator and the error bars represent standard deviation within a mix composition/operator 


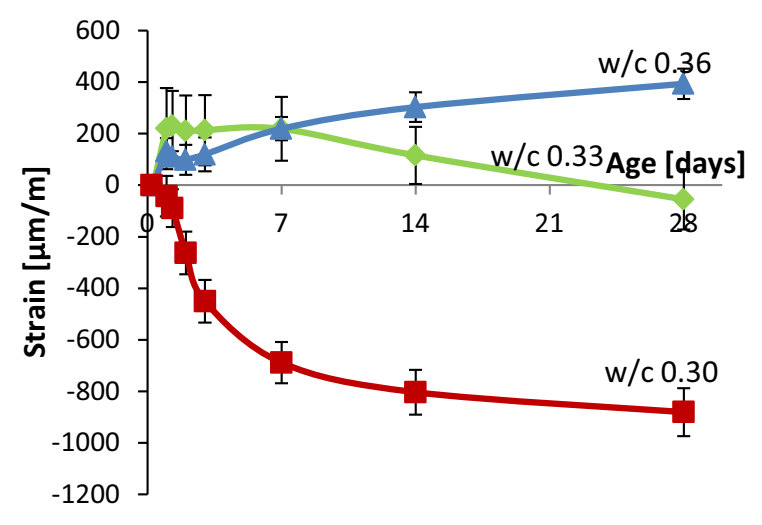

Fig. 3 Autogenous deformations of cement pastes according to standard measurements obtained as the average from the four operators (averaging individual curves from Fig. 1). The error bars represent the standard deviation obtained from the sets of averaged results from each operator

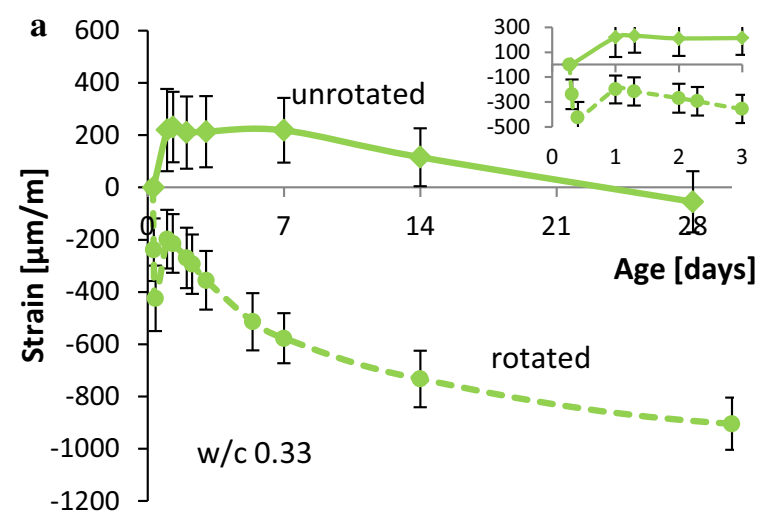

In Fig. 4, the results of the samples rotated from casting until final set (measurements run only by one operator) are compared to the standard measurements without rotation (results from all operators from Fig. 3). The highest difference was found for the w/c 0.33 cement paste. It needs to be also noted that bleeding was visually evident only for this cement paste. The fact that bleeding occurred in the pastes at w/c 0.33, while it was not evident for the pastes of higher w/c 0.36 was likely due to a lower workability of the w/c 0.36 paste brought about by the higher SAP amount. This allowed avoiding segregation of the mixture and therefore bleeding. For the w/c 0.33 paste, unrotated (bleeding) samples show high initial expansion and shrinkage starts only at approximately 7 days. For the other two mixes, the curves show a

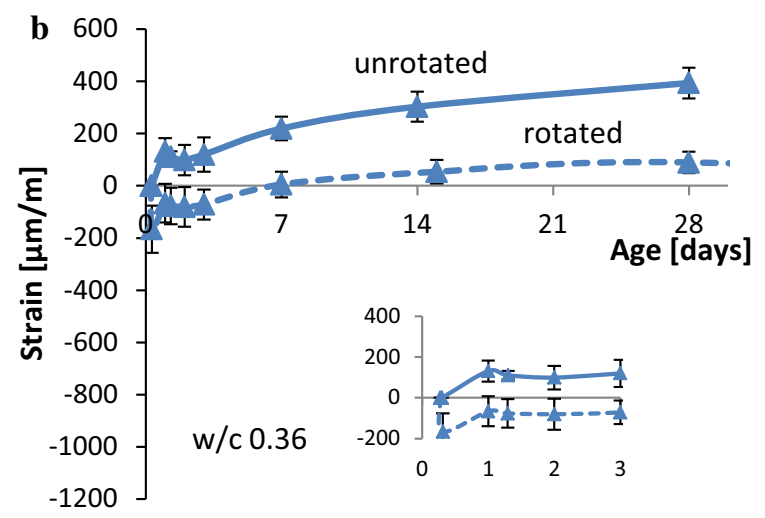

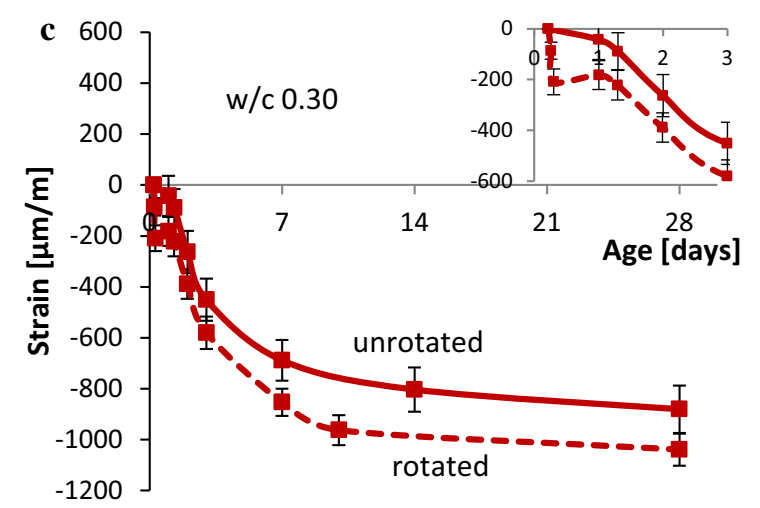

Fig. 4 Autogenous deformations of cement pastes (a w/c 0.33, b w/c 0.36, $\mathbf{c} \mathrm{w} / \mathrm{c} 0.30$ ) according to standard measurements on unrotated samples (average of 4 operators, the same set of results as in Fig. 3) and on samples rotated from casting until setting (results by one operator, error bars refer to standard deviation between individual samples) 
very similar trend except for the initial higher shrinkage observed on rotated samples, with the increase in shrinkage magnitude at the initial stages of deformation equal to about $200 \mu \mathrm{m} / \mathrm{m}$.

\subsection{Automatic measurements}

At the time of Vicat-determined final set, the temperature of the w/c 0.30 cement paste inside the corrugated tubes placed in the oil bath next to the samples used in the automatic measurements was $20.0 \pm 0.1{ }^{\circ} \mathrm{C}$. The maximum temperature was $20.8 \pm 0.1{ }^{\circ} \mathrm{C}$ and was recorded at approximately $12 \mathrm{~h}$ from mixing. The maximum temperature difference between the surrounding oil and the samples was equal to $0.6{ }^{\circ} \mathrm{C}$.

In Fig. 5, automatic measurements (data from one mixing by one operator for each mix composition) are presented and compared to standard measurements (the same results as in Fig. 3) for a period of 7 days. Automatic measurements are presented as average from the three measuring stations (on which three samples from the same mixing were measured), with standard deviation indicated only at chosen time points (to enable comparison with the standard measurements). The low scatter of the automatic measurements is also evident in Fig. 5.

It can be seen that the results agree very well between the automatic measurements and standard

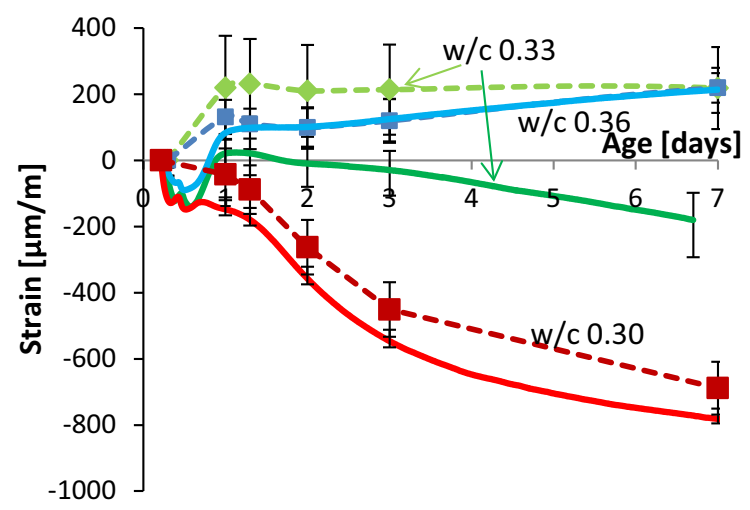

Fig. 5 Autogenous deformations of cement pastes according to automatic measurements (continuous lines) and standard measurements (dashed lines with markers). The results are the average from three samples prepared by one operator (automatic) and from four operators (standard). The error bars represent the standard deviation (shown only at chosen time points for the automatic measurements) measurements for w/c 0.30 and w/c 0.36 cement pastes, both regarding the trends and the determined values. For the w/c 0.33 paste, the disagreement in the values could be due to the shift resulting from initial measurement, similarly as in the case of the scatter visible in Fig. 2c, d. However, a different trend was visible also in the period between 3 and 7 days, with automatic measurements showing moderate shrinkage, while hardly any shrinkage was observed on average with the standard measurements. At the same time, the w/c 0.33 cement paste showed the highest scatter between different operators in the standard measurements, and between different samples even in the automatic measurements. The reason for the disagreement between the two groups of measurements is most probably due to the effect of bleeding (see below in the discussion section) influenced by the different temperature histories of the samples from automatic measurements stored in the bath and standard measurements stored in the air.

\subsection{Statistical data assessment}

The findings of the repeated measurements ANOVA were as follows:

- According to all analysed models (see Eqs. 1-3 above), there is a statistically significant difference considering the factor mix composition. This means that the method is sensitive enough to follow differences between the different tested mix compositions. However, this is only the case when all compositions are considered. When only w/c 0.33 and w/c 0.36 compositions are considered, no statistically significant difference exists.

- According to all tested models, the factor age is statistically highly significant (i.e. the evolution of shrinkage in time can be followed by the method).

- The interaction between age and mix composition is statistically highly significant (i.e. the different courses in time of the shrinkage of different mix compositions can be followed by the method).

- The effect of operator is not statistically significant $(p$ value $=0.12)(\operatorname{model} 3)$.

It should be noted that the significance is set at 0.05 level. For the significant factors, the $p$ values were always considerably lower, below 0.01 .

Further steps of the statistical assessment considered estimation of precision of the standard method 
according to the ISO 5725-2 standard [22]. The pooled within-operator standard deviation obtained based on independent specimens treated as replicates was equal to $s_{\mathrm{r}}=129 \mu \mathrm{m} / \mathrm{m}$. The between-operator standard deviation was equal to $s_{\mathrm{O}}=81 \mu \mathrm{m} / \mathrm{m}$. Finally, the between-operator reproducibility standard deviation was equal to $s_{\mathrm{OR}}=152 \mu \mathrm{m} / \mathrm{m}$.

As a comparison, the pooled within-operator standard deviation from automatic measurements (calculated from the three mixes tested by one operator, presented in Fig. 5) was $s_{\mathrm{r}}=56 \mu \mathrm{m} / \mathrm{m}$.

\section{Discussion}

\subsection{Effect of time-zero measurement}

As already mentioned in Sect. 2.1, the main reason for the within-operator discrepancies between the individual samples measured by each operator was due to the differences in the initial measurement at final setting time (time-zero measurement). This problem has been discussed in detail in e.g. [15, 18, 19]. Interestingly, after averaging the single samples' result within each operator, the differences between different specimens within an operator seem to compensate each other and the scatter between different operators becomes much lower (compare scatter in Fig. 2c, d with that in Fig. 3). Two reasons may be responsible here for the high scatter: (1) the determination of setting time, including both systematic effect and random error, and (2) random error on the initial length measurement at time zero.

If one considers that the temperature evolution in the relatively bulky samples used for determining the setting time with the Vicat needle leads to slightly accelerated hydration compared to the paste in the corrugated tubes, the consequence should be that the paste in corrugated tubes sets in fact only after timezero determined with the Vicat method. This leads to a situation in which the deformation measurements necessarily contain strains occurring before final set of the samples in the corrugated tubes. This is therefore supposed to be a systematic effect, leading consequently to an increase in the measured strain. In order to avoid the temperature increase in the Vicat samples, steel moulds could be used, possibly conditioned at constant temperature by means of water circulation in the casing around the moulds.
The systematic error due to a difference in setting time occurring between the Vicat samples and the corrugated tubes can be estimated as follows. Considering that the strain rate in the \pm 15 min time window around the setting time determined with Vicat needle changes between 1.5 and $2.5 \mu \mathrm{m} / \mathrm{m} / \mathrm{min}$ (for w/c 0.30 cement paste), a difference of $30 \mathrm{~min}$ should result in approximately $60 \mu \mathrm{m} / \mathrm{m}$ difference in the determined strains.

Different methods can be used to determine timezero alternative to the Vicat needle penetration test, e.g. acoustic emission [32], elastic modulus evolution with ambient response method [33], rheology tests or measurements of restrained deformations [34] (see also [17]). The methods based directly on measurements of deformation seem most appropriate for excluding the initial steep part of the shrinkage that is not corresponding to a build-up of internal stresses. Time-zero has been identified in $[15,16]$ as the time instant at which the shrinkage rate changes drastically. It needs to be noted that such methods are disputable if there are periods of expansion preceded and followed by shrinkage around setting time, where it is hard to define a single change in shrinkage rate.

In addition to the systematic error leading to a higher measured shrinkage, starting the measurements too early will also increase the scatter, since high scatter is a characteristic of deformation measurements performed before final set, as also discussed in [11].

Based on this observation, time-zero can be determined as a time instant when the scatter of the deformation rates of replicate samples reaches constant low level. This procedure leads to determining time-zero approximately $6 \mathrm{~h}$ later than that determined as setting time for the pastes studied here. When it is applied for the automatic tests performed by one operator on three mix composition (Fig. 5), the polled within-operator standard deviation reduces from $s_{\mathrm{r}}=56 \mu \mathrm{m} / \mathrm{m}$ to $s_{\mathrm{r}}=21 \mu \mathrm{m} / \mathrm{m}$.

Another important source of random error manifesting in the scatter between the individual specimens is due to the fact that the sample is very compliant before or shortly after final set and therefore the length can change easily when placing the sample on the measuring bench or positioning the tip of the displacement transducer at the end plug. Finally, measurements started too early may lead to damaging of the samples and may even necessitate discarding the 
specimen. Even more detrimental is the case when a specimen is cracked but this is not noticed by the operator, leading to false results. The errors due to initial length measurement could be possibly minimized if the sample is not moved at least until the first measurement, which takes place in automatic measurements. All automatic measurements were performed by the same operator, with the polled withinoperator standard deviation equal to $s_{\mathrm{r}}=56 \mu \mathrm{m} / \mathrm{m}$. This is considerably less than the value calculated for all operators from standard measurements, $s_{\mathrm{r}}=129$ $\mu \mathrm{m} / \mathrm{m}$. However, the experienced operator running the automatic measurements obtained also considerably lower scatter in standard measurements, $s_{\mathrm{r}}=50 \mu \mathrm{m} /$ $\mathrm{m}$ (obtained from 3 mixes without rotation and 3 mixes with rotation run by that operator). Since the repeatability standard deviation was very similar for both automatic and standard measurements, it can be concluded that sufficiently delicate handling of the samples as provided by an experienced operator should allow avoiding the scatter due to initial measured length.

Another possible way of minimizing the scatter, fully available if only standard measurements are at hand, is to use the Vicat-determined setting time, followed by normalizing the curves from individual specimens to the average length at the end of the measurement. This approach seems justified considering that the overall magnitude of determined average shrinkage experiences relatively little scatter, even between the different operators, (see Fig. 3).

Such procedure allows reducing the polled withinoperator standard deviation (repeatability precision) from $s_{\mathrm{r}}=129 \mu \mathrm{m} / \mathrm{m}$ to $s_{\mathrm{r}}=61 \mu \mathrm{m} / \mathrm{m}$, and reducing the between-operator reproducibility standard deviation from $s_{\mathrm{OR}}=152 \mu \mathrm{m} / \mathrm{m}$ to $s_{\mathrm{OR}}=102 \mu \mathrm{m} / \mathrm{m}$.

\subsection{Effect of rotation}

The effect of samples rotation before final set is shown in Fig. 4 for the standard measurements. It needs to be noted that bleeding was evident only in the samples of the w/c 0.33 paste. In this case, avoiding bleeding with rotation of the samples led to considerable changes in the shrinkage behaviour during the first 7 days of hydration. If no rotation is provided and bleeding is allowed to take place, reabsorption of bleeding water causes hygral swelling of the cement paste [24, 35]. This was mentioned as a possible interference also in the standard [13], however it was suggested that the effect should be negligible for cement pastes or mortars of w/c lower than 0.40 . If one considers that the measured autogenous deformation is the net effect of the competition between swelling and autogenous shrinkage (as discussed e.g. in [35, 36]), rotation may alter the results even if no visible bleeding exists. This can be also concluded from [24], where it was observed that rotation altered not only the initial swelling behaviour, but also the further course of the shrinkage curves. Unlike hypothesized in the standard [13], the effect of rotation on increasing initial shrinkage or reducing initial expansion may affect the results also in low w/c cement pastes without internal curing, see the results shifted for the w/c 0.30 cement paste, Fig. 4c.

On the other hand, considering that the real set in the corrugated tubes takes place later than that determined with the Vicat method, the dependence of the shrinkage upon rotation occurs in great part in the phase where the paste is not set yet. Therefore, the effect of rotation in non-bleeding pastes could be in great part avoided if the time-zero was appropriately determined later than indicated by the Vicat test, as discussed in Sect. 4.1.

One issue that has not been sufficiently studied in the literature is the possible influence of rotation on the microstructure, even excluding the effect of bleeding. It may be possible that rotation of the sample before setting disturbs formation of solid bonds and therefore offsets setting. It is also worth mentioning that the difference between rotated and unrotated samples increased when rotation speed was increased to $9 \mathrm{rpm}$, considerably higher than 1-3 rpm prescribed by the standard and $2 \mathrm{rpm}$ used in all other tests here. This was studied with automatic measurements for the w/c 0.30 cement paste. With the increased rotation speed, the higher shrinkage in rotated samples continued for about $2.5 \mathrm{~h}$ after the nominal time-zero and the increase in shrinkage magnitude due to rotation reached as high as about $500 \mu \mathrm{m} / \mathrm{m}$.

\subsection{Effect of temperature}

The temperature evolution in corrugated tubes filled with w/c 0.30 cement paste and stored in air shows that the temperature effects on deformation need to be considered with care, even if a sample is stored in controlled climate conditions. Considering the 
measured temperature increase of $3.5^{\circ} \mathrm{C}$ and the coefficient of thermal expansion reported in [37] for a cement paste of the same w/c equal to $16 \mu \mathrm{m} / \mathrm{m} /{ }^{\circ} \mathrm{C}$ at the age of $15 \mathrm{~h}$ (when the temperature peak was observed in the air-conditioned samples), the contribution of thermal expansion/contraction to the measured deformation may be $50 \mu \mathrm{m} / \mathrm{m}$ or higher. When the samples are conditioned in the oil bath with forced oil circulation, heat dissipation from the samples is much more efficient and the effect of temperature in the samples becomes negligible.

Another temperature effect regards the difference in temperature between the samples used for Vicat tests and the corrugated tubes. As already discussed in Sect. 4.1, temperature increase in Vicat samples will likely lead to faster setting than in the corrugated tubes.

Knowing the evolution of temperature in both types of samples, the difference in setting time can be estimated based on equivalent age concept, e.g. [38]. For this purpose, isothermal calorimetry run at $20{ }^{\circ} \mathrm{C}$ (results not presented here) was used as a reference hydration heat evolution. The activation energy was assumed after [39] as equal to $45.4 \mathrm{~kJ} / \mathrm{mol}$. Based on the assumption that final set occurs at the same degree of hydration or maturity at different temperatures, it was estimated that final set occurs approximately 28 and 34 min later than in the Vicat test for the samples inside the corrugated tubes stored in the air and in the oil bath, respectively. As estimated in Sect. 4.1, such delay will lead to a considerable increase in the measured shrinkage by about $50-60 \mu \mathrm{m} / \mathrm{m}$ and will further provoke random errors on the measurements around the time-zero.

\subsection{Statistical method assessment}

An important observation regards the apparent independence of the results variability from the level of measured strain. Considering the precision of the measurements at a level sometimes similar to the measured strain, this may lead to problems in evaluating the results for less shrinking samples.

The determined precision at a level of about 130 and $150 \mu \mathrm{m} / \mathrm{m}$ for repeatability and between-operator reproducibility, respectively, fairly agrees with the preliminary estimate given in the standard, where pooled one-operator standard deviation was indicated as equal to $130 \mu \mathrm{m} / \mathrm{m}$. The precision can be further enhanced by applying normalization of the curves which allows reducing the error due to initial length measurement, or adjusting the time-zero as discussed in the previous sections.

\section{Conclusions}

In this paper, an experimental investigation on autogenous shrinkage measurements was performed with three different cement pastes measured in accordance with the standard ASTMC1698-09 by four operators. Additionally, automatic measurements were performed and the results were compared with standard measurements. The scatter between average shrinkage values measured by different operators was reasonably low, while very high scatter was found between the individual replicate specimens of the same mix composition if the operator was not experienced in using the method. The main source of scatter was due to the inaccuracies in initial length measurements, so called time-zero measurements. The random component of the error at time-zero measurement was mainly due to the affected initial sample length at handling the soft samples and the high scatter of the deformation in the plastic stage. The random component of the error can be reduced down to the level obtained in automatic measurements (where the samples are not touched and remain intact during the measurement) if sufficient care is taken during handling the samples as evidenced by the tests run by an experienced operator. The polled within-operator standard deviation (deviation between replicate samples from the same mix) was equal to about $130 \mu \mathrm{m} / \mathrm{m}$, while the between-operator reproducibility standard deviation was equal to about $150 \mu \mathrm{m} / \mathrm{m}$. The highest precision obtained by a single operator in both automatic and standard measurements corresponded to standard deviation of about $50 \mu \mathrm{m} / \mathrm{m}$.

Further care should be given at maintaining the samples at constant temperature. It was found that airstorage may not always allow for maintaining constant temperature in a hydrating cement paste. Further, considerably higher temperature was developing in the bulky Vicat samples used for determining timezero compared to the thinner samples inside the corrugated tubes. This led to slower hydration of the cement pastes in the corrugated tubes and consequently delayed setting (by about $30 \mathrm{~min}$ ) compared to that occurring in Vicat samples. Another factor that 
was studied was rotation of the samples, which is often employed to reduce bleeding. Rotation leads to higher observed shrinkage not only in the cement pastes were visible bleeding occurred, but can also affect the initial shrinkage in the cement pastes with w/c as low as 0.30 . The latter effect may however in large part take place before the actual final set of the cement pastes in the corrugated tubes.

Acknowledgments We would like to thank Mr Stephan Geis (Empa) for his help in running part of the measurements. The financial support for Zhangli $\mathrm{Hu}$ was provided by China Scholarship Council (File No. 201306130062).

\section{References}

1. Tazawa E-I, Miyazawa S (1995) Experimental study on mechanism of autogenous shrinkage of concrete. Cem Concr Res 25:1633-1638

2. Jensen OM, Hansen PF (2001) Autogenous deformation and RH-change in perspective. Cem Concr Res 31:1859-1865

3. Lura P, Jensen O (2007) Measuring techniques for autogenous strain of cement paste. Mater Struct 40:431-440

4. Jensen OM, Hansen PF (1996) Autogenous deformation and change of the relative humidity in silica fume-modified cement paste. ACI Mater J 93:539-543

5. Snoeck D, Jensen OM, De Belie N (2015) The influence of superabsorbent polymers on the autogenous shrinkage properties of cement pastes with supplementary cementitious materials. Cem Concr Res 74:59-67

6. Kovler K, Zhutovsky S (2006) Overview and Future Trends of Shrinkage Research. Mater Struct 39:827-847

7. Lura P, Jensen OM, van Breugel K (2003) Autogenous shrinkage in high-performance cement paste: an evaluation of basic mechanisms. Cem Concr Res 33:223-232

8. Sant G, Lura P, Weiss J (2006) Measurement of volume change in cementitious materials at early ages: review of testing protocols and interpretation of results transportation research record. J Trans Res Board 1979(2006):21-29

9. Bouasker M, Mounanga P, Khelidj A, Coué R (2008) Free autogenous strain of early-age cement paste: metrological development and critical analysis. Adv Cem Res 20:75-84

10. Bjøntegaard $\varnothing$, Budelmann H, Krauss M, Martius-Hammer TA (2015) RILEM Technical Committee 195-DTD Recommendation for test methods for AD and TD of Early Age Concrete, Springer

11. Jensen OM, Freiesleben Hansen P (1995) Hansen, A dilatometer for measuring autogenous deformation in hardening portland cement paste. Mater Struct 28:406-409

12. Gao P, Zhang T, Luo R, Wei J, Yu Q (2014) Improvement of autogenous shrinkage measurement for cement paste at very early age: corrugated tube method using non-contact sensors. Constr Build Mater 55:57-62

13. ASTM (2009) ASTM C1698-09 Standard test method for autogenous strain of cement paste and mortar, ASTM International, West Conshohocken, PA
14. Bao Y, Meng W, Chen Y, Chen G, Khayat KH (2015) Measuring mortar shrinkage and cracking by pulse prepump Brillouin optical time domain analysis with a single optical fiber. Mater Lett 145:344-346

15. Sant G, Lura P, Weiss J (2006) A discussion of analysis approaches for determining 'time-zero'from chemical shrinkage and autogenous strain measurements in cement paste. In: Jensen O, Lura P, Kovler K (eds) International RILEM conference on volume changes of hardening concrete: testing and mitigation., hardening concrete: testing and mitigationRILEM Publications SARL, Bagneux, pp 375-383

16. Seddik M, Meddah A (2011) Tagnit-Hamou, evaluation of rate of deformation for early-age concrete shrinkage analysis and time zero determination. J Mater Civ Eng 23:1076-1086

17. Liu J-P, Tian Q, Sun W, Miao CW, Tang MS (2006) Study on the self-desiccation effect in early-age concrete and the determination of "time-zero" of self-desiccation shrinkage. In: Jensen O, Lura P, Kovler K (eds) International RILEM conference on volume changes of hardening concrete: testing and mitigation. RILEM Publications SARL, Bagneux, pp 401-410

18. Kovler K (2007) Effects of internal curing on autogenous deformation. In: Kovler K, Jensen O (eds) Report rep041: internal curing of concrete, state-of-the-art report of RILEM technical committee 196-ICC, pp 71-104

19. Weiss J (2003) Experimental determination of the'Time Zero', t0 ('Maturity-Zero', M0). In: Bentur A. (ed) Early age cracking in cementitious systems-report of RILEM technical committee 181-EAS-Early age shrinkage induced stresses and cracking in cementitious systems, pp 195-206

20. Tian Q, Jensen OM (2009) Measurement with corrugated tubes of early-age autogenous shrinkage of cement-based material. Guisuanyan Xuebao 37:39-45

21. Crowder MJ, Hand DJ (1990) Analysis of repeated measures. CRC Press, London

22. ISO 5725-2 (2002) Accuracy (trueness and precision) of measurement methods and results-Part 2: basic method for the determination of repeatability and reproducibility of a standard measurement method (12.02)

23. Jensen OM, Hansen PF (2001) Water-entrained cementbased materials: I. principles and theoretical background. Cem Concr Res 31:647-654

24. Mohr BJ, Hood KL (2010) Influence of bleed water reabsorption on cement paste autogenous deformation. Cem Concr Res 40:220-225

25. Justs J, Wyrzykowski M, Bajare D, Lura P (2015) Internal curing by superabsorbent polymers in ultra-high performance concrete. Cem Concr Res 76:82-90

26. ASTM (2013) ASTM C191-13 standard test methods for time of setting of hydraulic cement by Vicat Needle, ASTM International, West Conshohocken

27. Oehlert GW (2000) A first course in design and analysis of experiments. WH Freeman New York, New York

28. Verbeck GJ, Helmuth RH (1968) Structures and physical properties of cement paste, proceedings of the 5th international symposium on the chemistry of cement, pp 1-32

29. Jensen OM, Hansen PF (2002) Water-entrained cementbased materials II. Experimental observations. Cem Concr Res 32:973-978 
30. Mechtcherine V et al (2014) Effect of internal curing by using superabsorbent polymers (SAP) on autogenous shrinkage and other properties of a high-performance finegrained concrete: results of a RILEM round-robin test. Mater Struct 47:541-562

31. Lura P, Durand F, Jensen OM (2006) Autogenous strain of cement pastes with superabsorbent polymers. In: Jensen O, Lura P, Kovler K (eds) International RILEM conference on volume changes of hardening concrete: testing and mitigation. RILEM Publications SARL, Bagneux, pp 57-65

32. Couch J, Lura P, Jensen OM, Weiss J (2006) Use of acoustic emission to detect cavitation and solidification (time zero) in cement pastes. In: Jensen O, Lura P, Kovler K (eds) International RILEM conference on volume changes of hardening concrete: testing and mitigation. RILEM Publications SARL, Bagneux, pp 393-400

33. Maia L, Azenha M, Faria R, Figueiras J (2012) Identification of the percolation threshold in cementitious pastes by monitoring the E-modulus evolution. Cem Concr Compos 34:739-745
34. Sant G et al (2009) Detecting the fluid-to-solid transition in cement pastes. Concr Int 31:53-58

35. Bjøntegaard $\varnothing$, Hammer TA, Sellevold EJ (2004) On the measurement of free deformation of early age cement paste and concrete. Cem Concr Compos 26:427-435

36. Barcelo L, Moranville M, Clavaud B (2005) Autogenous shrinkage of concrete: a balance between autogenous swelling and self-desiccation. Cem Concr Res 35:177-183

37. Wyrzykowski M, Lura P (2013) Moisture dependence of thermal expansion in cement-based materials at early ages. Cem Concr Res 53:25-35

38. Pang X, Bentz DP, Meyer C (2012) Modeling cement hydration kinetics using the equivalent age concept, ultrahigh performance concrete and nanotechnology in construction. Proceedings of Hipermat 2012. 3rd international symposium on UHPC and nanotechnology for high performance construction materials, Kassel University Press $\mathrm{GmbH}$

39. Chen H (2013) Autogenous and thermal deformations and their interaction in early age cementitous materials. EPFL, PhD Thesis, Lausanne 